\title{
VECTOR: An IP-based Remote Combat Robot
}

\author{
A. S. Zaforullah Momtaz \\ University of Asia Pacific \\ Dhaka, Bangladesh
}

\author{
Molla Rashied Hussein \\ University of Asia Pacific \\ Dhaka, Bangladesh
}

\author{
Nahida Sultana \\ Chowdhury \\ University of Asia Pacific \\ Dhaka, Bangladesh
}

\begin{abstract}
In this paper, an IP (Internet Protocol) -based Remote Combat Robot is conversed, namely VECTOR (Vigorous Efficient Combatant Tactical Operative Robot). The name VECTOR is chosen to depict the concrete physiognomies of the Robot which is assembled. This project is designed and developed to operate remote combat. IP communication is enforced to control every single state of VECTOR. Advantage over other system is that VECTOR can rotate up to $180^{\circ}$ angle with respect to the 2-D axes both in clockwise and counter clockwise direction as per need. Moreover, the other prospects of this project are that VECTOR can be deployed to carry out Armored Hefty Freight, Rescue Mission during Natural Calamities, Safekeeping Tasks, Social Services and many more to name.
\end{abstract}

\section{Keywords}

Robot, Internet Protocol, Remote Combat, LASER Gun, Machine Gun, Double Barrel Turret.

\section{INTRODUCTION}

The word Combat derived firstly from the Middle French (1535-45) word Combattre and subsequently Late Latin [1] word Combattere which is equivalent to Latin word Com (To) and the Latin word battuere (strike, beat). The word Combat became ubiquitous when it is used to denote the phenomenal French Resistance formed in the non-occupied region of France during the World War II (1939-1945).

Remote Combat is a combat technique that involves sending machine to a distant place and controls that machine to do perform combat operation from a safer place. That mentioned machine could be Robots, Unmanned Airplanes/ Helicopters/ Battleships/ Tanks and more to come. However, the scope of this paper is limited solitary to the remote combat of Robots.

The word Robot, which is a Czech word meaning Worker, was first used in a 1921 play titled R.U.R. Rossum's Universal Robots, by Czechoslovakian writer Karel Capek. MerriamWebster describes robot as "A machine that looks like a human being and perform various complex acts; a device that automatically performs complicated, often repetitive tasks; a mechanism guided by automatic controls." International Organization for Standardization (ISO) describes a robot as "An automatically controlled reprogrammable, multipurpose manipulator programmable in three or more axes, which may be either fixed in place or mobile for use in industrial automation applications"[2].

Internet Protocol, commonly referred as IP, is a suite of Protocols with the articulated seek of granting meaningful computer communications. It was initially built up for the United States Department of Defense Advanced Research Projects Agency (DARPA) network. Now it is used to link private, commercial, educational and military organizations across the world. Due to its flexibility, it has become the defacto networking standard. Even though it lacks an integral error reporting mechanism, it subsidizes by relying on upper layer protocols. Internet Control Message Protocol (ICMP) provides low level reporting and consequently becomes integral part of IP [3].

This project aims to represent a robot, performing remote combat to avoid physical presence of human being, termed as VECTOR. In VECTOR, it includes two (Double) Barrel Turrets (Left and Right), Machine Gun, Laser Gun and others. For movement and balance purpose, four Wheels and two Engines are assembled. The reason behind these two Engines is to rotate VECTOR in $180^{\circ}$ angle with respect to the 2-D axes both in clockwise and counterclockwise direction as per requirement.

In the web interface of this project actions are assigned to Left Barrel Turret, Right Barrel Turret, Machine Gun, Motion button and Angle setter. Using IP-based interface, one click quick action is implemented. Joystick is playing the vital role of the controller, as by pressing Joystick's buttons, user can regulate the VECTOR through IP. The system structure is presented in Fig. 1.

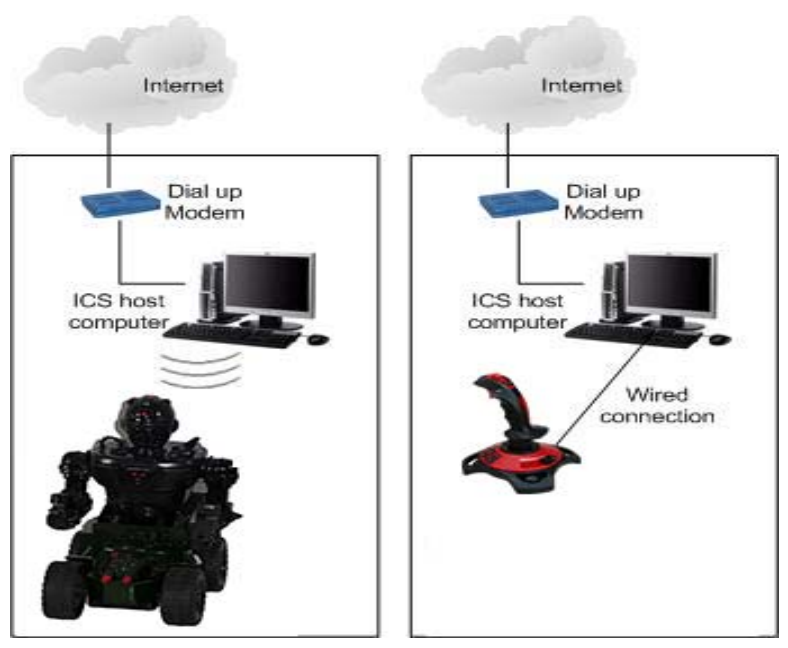

Fig 1. System Structure

The organization of this paper is as follows: in Section 2, the Literature Review is narrated, Section 3 describes the detail design of VECTOR with subsections of hardware and software implementation, Section 5 shows the result of the work being done. Finally, Section 6 draws out the conclusion with some suggestions on the future work to be pursued.

\section{LITERATURE REVIEW}

For Developing VECTOR, initially, [4] have been gone through, where developing a robotic vehicle have been learnt about using RF technology for remote operation attached with wireless camera for monitoring purpose. That robot along with camera can wirelessly transmit real time video with night vision capabilities. That is kind of robot can be helpful for spying purpose in war fields. An 8051 series of Microcontroller is used for the desired operation. At the transmitting end using push buttons, commands are sent to the 
receiver to control the movement of that robot either to move forward, backward and left or right etc. That Combat robot is radio operated, it has got two barrel turret through bullet can be fired, radio camera in synchronization with the turret can rotate up and down, left and right up to a safe firing limit turret and camera mechanism has been installed which has all the function like tank, turning to any angle on its axis, moving forward and reverse turning left and right, running instantly into reverse direction. The RF transmitter acts as a RF remote control. It has another function like a self suicide. Secondly, [5] and [6] have been meticulously gone through, where minimizing human casualties in terrorist attack was discussed. The combat robot in that paper has been designed to tackle such a cruel terror attacks. That robot is radio operated; selfpowered, and has all the controls like a normal car. A wireless camera has been installed on it, so that it can monitor enemy remotely when required. It can silently enter into enemy area and send us all the information through its' tiny Camera eyes. This spy robot can be used in Star Hotels, Shopping Malls, Jewellery Showrooms etc. where there can be threat from intruders or terrorists. Since human life is always precious, those robots are the replacement of fighters against terrorist in war areas. The robot in another paper has got two barrel turret through Bullet can be fired; Radio Camera in synchronization with the Turret can rotate up and down, Left and Right up to a safe firing limit. Turret and Camera Mechanism has been installed on the previous spy robot vehicle, which has all the function like tank, turning to any angle on its axis, moving forward and reverse turning Left and Right, running instantly into reverse direction. A pair of LASER Gun has been installed on it, so that it can fire on enemy remotely when required; this is not possible until a Wireless Camera is installed. Wireless camera will send real time video and audio signals which could be seen on a remote monitor and action can be taken accordingly. It can also silently enter into enemy area and send us all the information through its' tiny Camera eyes. It is designed for, fighting as well as suicide attack. Using the above references, the concepts for developing VECTOR are extracted.

\section{DESIGN OF VECTOR}

At this moment in time, Robotics plays the most vital role in making hard and toiling tasks easier in mundane existence. Moreover, the reliability of long distant communication has never been so upsurged since the beginning of Robotics era. Due to gradual increment of interest, Robotics is becoming essential part of daily life.

At present, Radio Frequency (RF) is mostly used as the primary mode for Robot communication. However, to create a RF network for long range of many simple applications is not a feasible solution. By keeping this in mind, IP-based communication is introduced to control the proposed Robot VECTOR.

System that installed in VECTOR is a combination of state of the art technology. Here, software plays the most important role in terms of decision making. The block diagram of VECTOR is represented in the Fig. 2.

In Fig. 2, Central Decision Unit (CDU) is supposed to take the all necessary action to operate all the sub-systems. Subsystem that installed inside the VECTOR is Wireless Transmission Module (WTM), Operation signal (OS), Machine Gun (MG), Vehicle Engine (VE), Double Barrel Turret (DBT) and LASER Gun (LG). WTM is supposed to do communication with the system commander. The uniqueness of WTM is that it is the only input module of the Robot. Pulse rate of OS represents working activities which in other term, the representative of emotions of this machine. MG is the hand held firing module and DBT is the other firing module for Cannonballs. LG is used for several thermal activities such as soldering and/or cutting. Moreover, it can also be used for LASER gun firing purposes. VE is the core machine which works as a chassis of the entire system. It has two electric motors which allow controlling each wheel pair separately. Therefore, the robot can rotate $180^{\circ}$ angle with respect to the 2-D axes both in clockwise and counter clockwise direction when situation demands. The CDU works as the brain of the robot and manages overall operations as well as takes appropriate actions instantaneously.

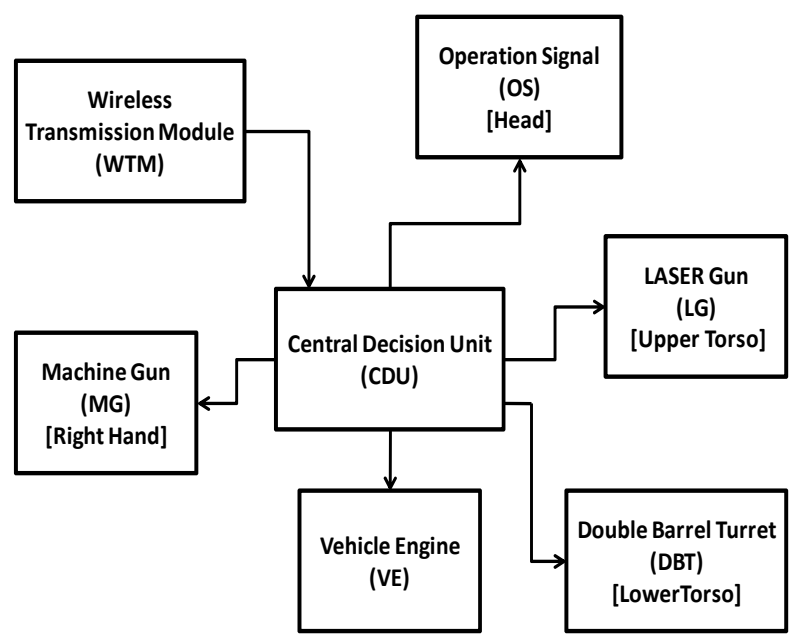

Fig 2. VECTOR System Block Diagram

The following sub-sections describe particulars about the Objectives and Transceiver (Transmitter and Receiver) implementation.

\subsection{Objectives}

The main objectives of using VECTOR are as follow:

1. Malignant Situation: Customarily, Robot is sent to work in fatal environment where human being should not be sent. It is because now the technology is available to prevent unnecessary lose of human life. Moreover, injury to Robot is very less significant than human injury. For this reason, it is a prudential judgement to send Robot instead of human in lethal task.

2. Rescue Mission: Sometimes, it is convenient for Robots to accomplish rescue mission due to lack of fatigue, lassitude or sluggishness. Robots do not get tired; they are slumbering deficient. They do not yawn, snooze or sleep. This attribute makes them a perfect match for monotonous, tedious, unexciting and repetitive job.

3. Warfare: In modern era, Robots are used in warfare to enhance accuracy and reduce stray injury of innocent persons. Although the warfare itself is destructive in nature, usage of Robots does not ensure demolition rather lessening inadvertent mutilation.

\subsection{Transceiver Implementation}

1. Components of Subsystem description: Heart of VECTOR is ATMEGA8, which is a Microcontroller from ATMEL Corporation. It responsible to decodes all the Commands received from the Transmitter and to execute Commands. The behaviours that are followed as follow: 
- Moves in Forward and Backward Direction

- $\quad$ Speed controls in both direction

- It can even rotate Left or Right while moving Forward or Backward Direction

- Forward running or instant reverse without stopping, as it could take turn on its axis.

The entire system is nothing but a prototype. That is why all the equipment is DC components. These are as follows:

i. ATMEGA8: An 8 Bit microcontroller is used of RISC architecture. It has $8 \mathrm{~KB}$ of Flash Memory, $1 \mathrm{~KB}$ of Static RAM, 512 Bytes of ROM and the operating speed is maximum $16 \mathrm{MHz}$. This Microcontroller has the total of $23 \mathrm{I} / \mathrm{O}$. Out of these lines, 11 lines are used as Output Channels and 4 lines are used as Input Channels [7].

ii. Wireless Module: This part is used to communicate with the base station. It acts as the Terminal of VECTOR. This module receives Commands and provides the update of that Commands.

iii. Power Supply: For the power backup, 3 AA size Battery cells $(+1.5$ Volts each) are used to provide +4.5 Volts. All the cells are rechargeable and Lithium-Ion in type.

iv. Barrels: Barrels are made of plastic body. In both Barrels, LEDs are used to indicate the firing state. Whenever a fire command is placed, respective LED will flash.

v. Engine: DC motors are the major motion creator. 2 DC motors are used to manage the movements of VECTOR. And these machines are parallel means the Right Wheels of VECTOR are connected with the Right Engine whereas the Left Wheels are connected with the Left Engine. Therefore, for a Forward Movement, both of the Engines will rotate in the same Forward direction and for Backward Movement, Engines will rotate in the same Backward direction. Moreover, in case of Left or Right turn, Engines will rotate in the Opposite directions, thus VECTOR can take its turn on its own axes.

vi. Machine Gun: In the Machine Gun, there is another LED used as the indicator of its fire. When a command is placed, Fire mode is activated then the corresponding LED is flashed.

vii. LASER Gun: LASER Gun is another firing option of VECTOR. Three LEDs are used here as the indicator. By the combination of these three LEDs, various Firing combinations can be produced.

viii. Head Indicator: This indicator is used to represent the emotion of VECTOR. It is symbolized by another LED. It is also used to demonstrate the progress of an assigned Task.

2. Software Implementation: For the software implementation, we deploy mikroC [8] software. It is a full-featured ANSI C compiler designed for 5 different microcontroller architectures. Therefore currently it becomes the finest solution for developing code in user favorite microcontroller. It features intuitive IDE, powerful compiler with advanced SSA optimizations, lots of hardware and software libraries, and additional tools that help to work. Target microcontroller architecture is ARM, which is why ATMEGA8 controller is used in the project.

\section{Flow charts for VECTOR Motion}

The flowchart is depicting the Robot Motion is shown in Fig. 3. It has been noticed that, at first, VECTOR receives a Controller Encrypted Input (CEI). Afterwards, that CEI gets decrypted using own customized decrypted method based on ASCII character and the Decrypted Instruction (DI) is pored over regarding its validity as a Motion Instruction (MI). If it is a MI, then further decisional breakdown is made. Otherwise, DI is discarded. If MI happens to be a Forward Motion Instruction (FMI), then VECTOR's Forward Motion is set. Likewise, If MI is Backward Motion Instruction (BMI) or Left Motion Instruction (LMI) or Right Motion Instruction (RMI), then VECTOR's backward or Left or Right Motion is set, respectively.

If MI happens to be a Forward Motion Instruction (FMI), then VECTOR's Forward Motion is set. Likewise, If $\mathrm{MI}$ is Backward Motion Instruction (BMI) or Left Motion Instruction (LMI) or Right Motion Instruction (RMI), then VECTOR's backward or Left or Right Motion is set, respectively.

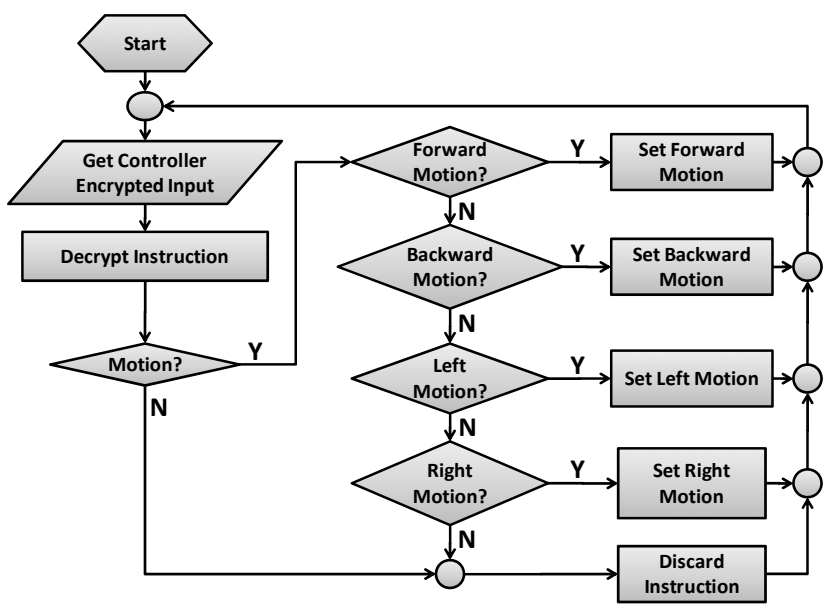

Fig 3. VECTOR Motion Flow Chart

\section{Flow charts for VECTOR Firing}

The flowchart is depicting the VECTOR firing is shown in Fig. 4. It has been observed that, at first, VECTOR receives a CEI and that CEI gets decrypted using own customized encrypted method based on ASCII character. The DDI is examined whether it is a valid as a Firing Instruction (FI) or not. If it is a FI, then further decisional breakdown is made. Otherwise, DI is discarded.

If FI happens to be a Machine Gun Instruction (MGI), then VECTOR brushfires. Likewise, If FI is LASER Instruction (LI) then VECTOR fires LASER. If FI is Double Barrel Turret Instruction (DBTI), then additional decisional breakdown is made. If DBTI is Left Turret Instruction (LTI) or Right Turret Instruction (RTI) or Both Turret Instruction (BTI), then VECTOR fires Left or Right or Both Turret, respectively. 


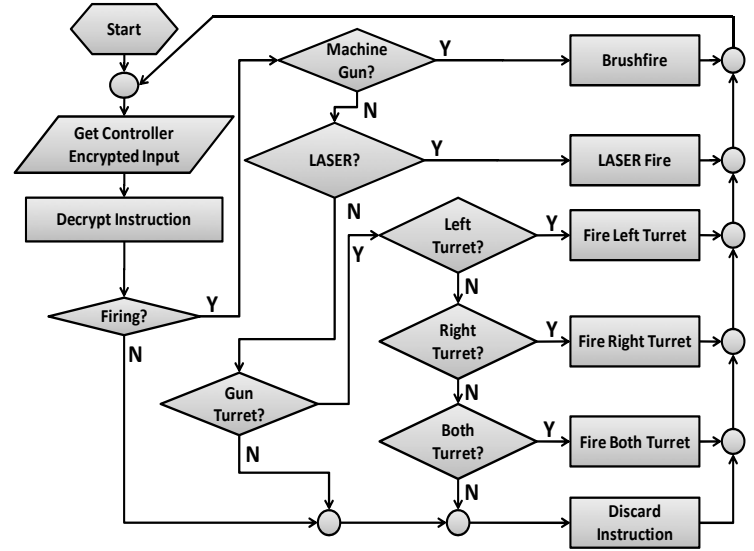

Fig 4. VECTOR Firing Flow Chart

\section{Pseudo code of the system}

Pseudo code for the whole system is separated into two parts, one for the VECTOR and other is for Controller.

\section{- $\quad$ Pseudo code for VECTOR}

$W O R D$, is the entire command generated by the controller RESPONSE, is an array, generated by VECTOR

Loop is infinite

CEI( ), is a function, Controller Encrypted Input: Reads the FM module for the input string which is encrypted and send by the commander. This function takes the input from FM module and returns the encrypted code word.

$D I($ ), is a function, Decrypt Input : translates the code ward to make it readable for VECTOR. This function takes the encrypted code word as input and returns the readable word for VECTOR.

MI( ), is a function, Motion Instruction : justify whether the command is motion or not. It takes the readable word as input and returns either TRUE or FALSE after justification.

SET_MOTION( ), is a function : it takes the readable word as input and affects the hardware according to the direction and speed.

FI( ), is a function, Fire Instruction : justify whether the command is fire or not. It takes the readable word as input and returns either TRUE or FALSE after justification.

SET_FIRE( ), is a function : it takes the readable word as input and affects the hardware according to the time (LASER) or amount of firing(Bullet/Cannon Ball).

MISS_CMD( ), is a function to justify the miss matched part of the commander.

$E O($ ), is a function: Encrypted Output. Takes the response as input and returns encrypted response.

$V E O($ ), is a function: VECTOR Encrypted Output. Affects FM module to send the response generated by VECTOR.

\section{VAR WORD}

VAR RESPONSE [ ]

WHILE (TRUE)

$W O R D=D I(C E I())$

IF MI (WORD ) == TRUE THEN

$$
\text { RESPONSE[0] = SET_MOTION(WORD) }
$$

END IF

IF FI( WORD ) THEN

RESPONSE[1] = SET_FIRE $($ WORD $)$

ELSE

$$
\text { RESPONSE[2] = MISS_CMD }(\text { WORD })
$$

END IF

$$
\operatorname{VEO}(\operatorname{EO}(\text { RESPONSE[] ) ) }
$$

\section{WEND}

- $\quad$ Pseudo code for Controller

$C E O$ (), is a function : takes the key input from joystick via USB. Takes the key press as input and returns the scan code.

$E I($ ), is a function : takes the scan code as input and returns encrypted word.

$C E O$ ( ), is a function : Commander Encrypted Output. Takes the word as input and affects the FM module to transmit the command.

VEI( ), is a function : VECTOR Encrypted Input. It receives the response from the FM module generated by VECTOR.

DI( ), is a function : Decrypted Input. Receives the encrypted code word as input and generates the readable word by the commander.

$\operatorname{DISPLAY(~),~is~a~function:~Generates~the~response~to~a~}$ graphical module (monitor) generated by VECTOR. Takes the response as input and produces graphical result.

WHILE ( TRUE )

CEO(EI(GET_KEY()))

$\operatorname{DISPLAY(DI(VEI())~)~}$

WEND

\section{RESULT ANALYSIS}

\subsection{Test Scenario of VECTOR}

In test scenario VECTOR is started to traverse from node A and the target is to reach at destination node G. The scenario graph is presented in fig. 5 . In this graph, single circle node represents intermediate node, double circles node represents destination node and rectangle represents the obstacle node. Sequence of traversing is as follows:

1. Arbitrarily place VECTOR at A

2. Rotate VECTOR towards B

3. Move forward VECTOR towards B

4. Move forward VECTOR D, during motion cut metal door using LASER

5. At $\mathrm{D}$, rotate $90^{\circ}$ in right

6. Move forward towards $\mathrm{E}$

7. Rotate $90^{\circ}$ in right with machine gun firing until rotation completed

8. Move towards F

9. Rotate $90^{\circ}$ in left 
10. Fire left barrel

11. Fire right barrel

12. Fire both barrels

13. Rotate $135^{\circ}$ in right

14. Move forward towards G (Goal)

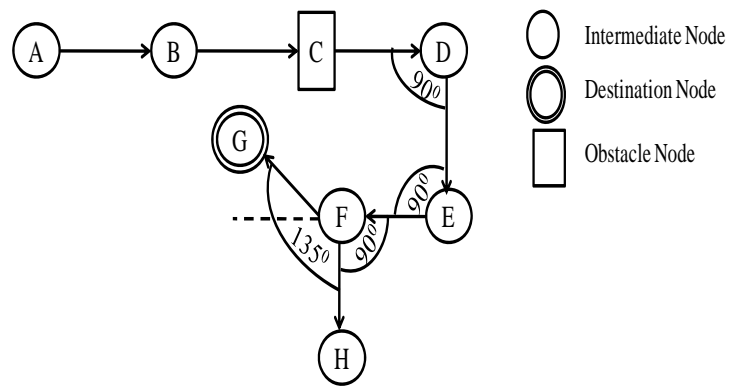

Fig 5: Test Scenario Graph

\subsection{VECTOR Activity Feature}

Feature of VECTOR is represented in table 1 with supported transformation criteria. All criteria that mentioned here are tested and satisfied the requirement of this project is started with.

Table 1. Activity Feature

\begin{tabular}{|c|c|c|c|c|c|c|}
\hline \multirow{2}{*}{\multicolumn{3}{|c|}{ Actions }} & \multicolumn{2}{|c|}{ Rotation } & \multicolumn{2}{|c|}{ Translation } \\
\hline & & & $\begin{array}{c}\text { CLW } \\
\text { (clockwise) }\end{array}$ & $\begin{array}{l}\text { CCW } \\
\text { (counter } \\
\text { clockwise) }\end{array}$ & Forward & Backward \\
\hline \multicolumn{3}{|c|}{ Motion } & $\sqrt{ }$ & $\sqrt{ }$ & $\sqrt{ }$ & $\sqrt{ }$ \\
\hline \multirow{5}{*}{ Firing } & \multicolumn{2}{|c|}{ Machine Gun } & $\sqrt{ }$ & $\sqrt{ }$ & $\sqrt{ }$ & $\mathrm{X}$ \\
\hline & \multicolumn{2}{|c|}{ LASER } & $\sqrt{ }$ & $\sqrt{ }$ & $\sqrt{ }$ & $\mathrm{X}$ \\
\hline & \multirow{3}{*}{ Barrel } & Left & $\sqrt{ }$ & $\sqrt{ }$ & $\sqrt{ }$ & $\mathrm{X}$ \\
\hline & & Right & $\sqrt{ }$ & $\sqrt{ }$ & $\sqrt{ }$ & $\mathrm{X}$ \\
\hline & & Both & $\sqrt{ }$ & $\sqrt{ }$ & $\sqrt{ }$ & $\mathrm{X}$ \\
\hline
\end{tabular}

\section{CONCLUSION AND FUTURE WORK}

In this paper, an IP-based Robot named VECTOR and its operations have been discussed. The prime feature of VECTOR is having the flexibility of rotating both in clockwise and counter-clockwise direction. VECTOR can fire while rotating. However, hands of VECTOR are nonrotatable. Thus, targeting something in angular upper or lower direction is complicated. Moreover, at present, VECTOR can take orders and executes those orders reactively.

In future, additional features will be added to allow rotation of the hands in angular upper or lower direction, as well as acting proactively, i.e. taking decisions as per situation, based on perceived states, by implementing Artificial Intelligence into VECTOR and turning into an Intelligent Agent. Current goal-based reflex decision making will be upgraded to utilitybased reflex. Optimality and Accuracy of VECTOR will be achieved during the next release of VECTOR.

\section{REFERENCES}

[1] Webster's Third New International Dictionary. "Late Latin". Volume II, H to R. Chicago: Encyclopædia Britannica, Inc. 1961.

[2] Lee,C.S.G., R.C. Gonzalez, K.S. Fu, Tutorials on Robotics, Computer Society Press, IEEE Computer Society, 1984.

[3] Miller, Philip, "TCP/IP: The Ultimate Protocol Guide", Universal-Publishers, 2009, pp. 3-5.

[4] Samad, Abdus, JadhavDevidasDasharath, and DhaigudeMadhukar Kumar. "An Intelligent Combat Robot", International Journal of Advanced Research in Computer and Communication Engineering (IJARCCE), Vol. 3, Issue 5, May 2014.

[5] Bhargavi, S., and S. Manjunath. "Design of an Intelligent Combat Robot for war fields." Department of Electronics and Communication Engineering, SJCIT, Chikballapur, Karnataka, India (2011).

[6] Singh, Abhinav Kumar, NilayaMitashShanker, and AnandPrakashYadav. "RF Controlled Terrorist fighting robot." International Journal of Computer Science \& Communication 1.1 (2010): 109-112.

[7] Official Webpage of ATmega8 - Atmel Corporation, Link: http://www.atmel.com/devices/atmega8.aspx, retrieved on 28 Feb 2015.

[8] MikroC Official Webpage, Link: http://www.mikroe.com/mikroc, retrieved on 28 Feb 2015. 